14 Chang YS, Liu CJ, Ou SM, et al. Tuberculosis infection in primary Sjögren's syndrome: a nationwide population-based study. Clin Rheumatol 2014; 33: 377-383.

15 Shang S, Ordway D, Henao-Tamayo M, et al. Cigarette smoke increases susceptibility to tuberculosis - evidence from in vivo and in vitro models. J Infect Dis 2011; 203: 1240-1248.

\title{
Suboptimal specificity of Xpert MTB/RIF among treatment-experienced patients
}

\section{To the Editor:}

The Xpert MTB/RIF assay (Cepheid, Sunnyvale, CA, USA) is strongly recommended by the World Health Organization as an initial diagnostic test for treatment-experienced patients of any retreatment category [1-3]. Yet, retreatment tuberculosis (TB) suspects have been infrequently included in studies of Xpert [4], probably because current-generation PCR-based tests are unable to determine Mycobacterium tuberculosis viability [5]. Indeed, Xpert is known to frequently remain positive at the end of standard short-course therapy [6], with case reports emerging of Xpert false-positivity for up to 5 years post-treatment completion [7, 8]. Furthermore, 56\% $(\mathrm{n}=3485 / 6285)$ of specificity data informing the most recent Cochrane meta-analysis [4] was derived from validation and demonstration studies [9, 10], which may be optimistic due to selection bias related to post-enrolment exclusions [7]. Not surprisingly, there have been increasing calls to clarify the guidelines for use of Xpert among treatment-experienced patients [11].

To address these concerns, we prospectively enrolled individuals with a history of prior treatment in a high HIV prevalence, limited resource setting (Harare, Zimbabwe) over a 2 year (2011-2013) period. We hypothesised that among individuals with a history of prior treatment, specificity would be lower than that reported in pooled summaries and would correlate with mean cycle threshold (i.e. mycobacterial load) and time since prior treatment completion. All participants provided written informed consent, and ethical approval was obtained from the Medical Research Council of Zimbabwe and the University of California, San Francisco Human Research Protection Program. Notified cases were categorised according to the outcome of their most recent course of treatment [1] as either 1) "recurrent TB" (TB following cure or completion of treatment of a previous TB episode), or 2) "prevalent retreatment TB" (treatment failure, which was defined as acid-fast bacilli sputum smear-positivity at month 5 or later). The reference standard for M. tuberculosis detection was a positive result on solid (Löwenstein-Jensen media), liquid (BBL MGIT Mycobacterial Growth Indicator Tubes (Becton Dickinson, Sparks, MD, USA)), or microscopic-observation drug-susceptibility (MODS) culture (TB MODS Kit; Hardy Diagnostics, Santa Maria, CA, USA) [12, 13]. Xpert false detection of active TB ("Xpert false-positive") was defined as Xpert-positivity in absence of any of the three culture modalities being positive. The Biomedical Research and Training Institute Tuberculosis Laboratory within the National Microbiology Reference Laboratory is a centre for Trials of Excellence in Southern Africa. The most recent Centre for American Pathologists assessment in 2014 demonstrated 100\% agreement for isoniazid, rifampicin, ethambutol and streptomycin resistance testing.

During the study, we enrolled 380 ambulatory retreatment TB cases, representing $\sim 65 \%$ of all retreatment TB cases notified to the Harare City Health Department during this time period. After excluding 37 patients who were not initiated on treatment, who were on second-line agents for known multidrug-resistant TB, or who did not have culture results available, 149 (43.4\%) patients had recurrent TB, and 194 (56.6\%) were prevalent retreatment cases. The diagnostic accuracy of Xpert for M. tuberculosis detection was evaluated among 149 patients with recurrent TB. Most (111 (74.5\%) out of 149) had HIV comorbidity with a median (interquartile range (IQR)) CD4 count of $177(83-350)$ cells $\cdot \mathrm{mm}^{-3}$. The median (IQR) time from completion of previous TB treatment to clinical re-presentation and Xpert testing was 19.6 (7.962.9) months. 127 (85.2\%) Xpert tests were generation 4. Of the 149 patients included in the analysis, 24 (16.1\%) had culture-positive rifampicin (RMP)-resistant TB; 65 (43.6\%) had culture-positive 
TABLE 1 Accuracy of Xpert MTB/RIF for Mycobacterium tuberculosis detection among patients with recurrent tuberculosis

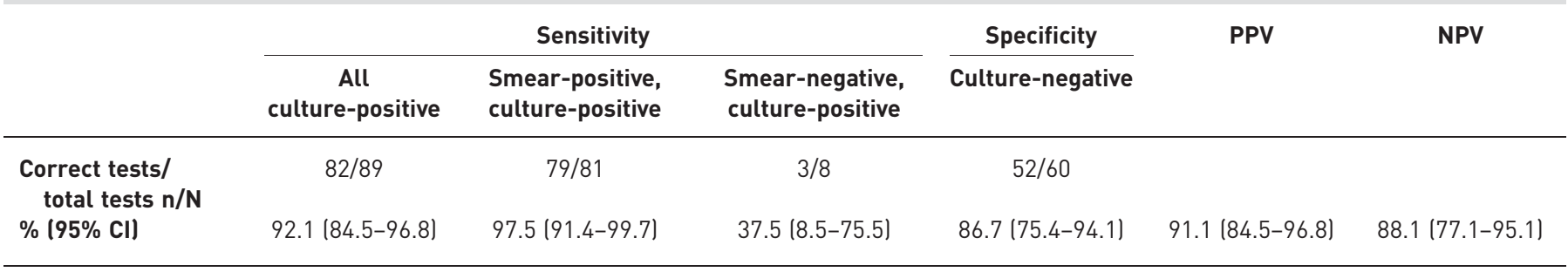

PPV: positive predictive value; NPV: negative predictive value.

RMP-susceptible TB; and 60 (40.2\%) had clinically defined TB. The overall sensitivity of Xpert was $92.1 \%$ (95\% CI 84.5-96.8\%; $\mathrm{n}=82 / 89$ ) (table 1). The sensitivity was $97.5 \%(95 \%$ CI $91.4-99.7 \% ; \mathrm{n}=79 / 81$ ) for smear- and culture-positive cases, and 37.5\% (95\% CI 8.5-75.5\%; $\mathrm{n}=3 / 8$ ) for smear-negative, culture-positive cases. The sensitivity of Xpert was not significantly affected by HIV status $(p=0.22)$. The false-positive rate among patients with recurrent TB was $13.3 \%$ (95\% CI 5.9-24.6\%). Mean \pm sD cycle threshold was lower for true- versus false-positive results $(21.3 \pm 5.0$ versus $28.0 \pm 5.3$ cycles, respectively; $\mathrm{p}<0.01)$. HIV co-infection was more common among individuals with false- $(100 \%)$ rather than true-positive $(69.5 \% ; \mathrm{p}=0.07)$ results. In multivariate analysis, mean cycle threshold independently predicted false detection of active TB $(\mathrm{p}<0.01)$, although time since prior TB treatment completion $(\mathrm{p}=0.58)$ did not. Model accuracy of mean cycle threshold alone was high (area under the curve (AUC) 0.82, 95\% CI 0.62-1.0), and each unit increase in cycle threshold (holding time since completion of prior TB treatment fixed) was associated with a $23.0 \%$ (95\% CI 6.0-42.0\%) increased relative risk of Xpert false-positivity.

Approximately 700000 cases of recurrent TB were notified in 2013 [1], with many times higher numbers presenting as recurrent $\mathrm{TB}$ suspects. Rapid treatment initiation in clinical practice and enrolment in clinical trials critically relies on the "rule-in" value of Xpert. Although meta-analyses have documented a high pooled specificity for M. tuberculosis detection (99\%, 95\% CI 90-100\%) [4], these pooled estimates included a small proportion of treatment-experienced patients. We found that among patients with recurrent $\mathrm{TB}$ (a history of prior $\mathrm{TB}$ treatment) up to one in seven can be expected to be Xpert false-positive for active TB. These results are similar to other studies where stratification of patients by history of prior TB treatment was possible [14]. In our study, higher relative mean cycle threshold predicted false-positivity, with values over 30 (Xpert quantitation result "very low") having a likelihood ratio of 5.4 and a specificity of $91 \%$ for false-positivity. However, these findings are preliminary, from a single centre, and should be examined in larger independently cross-validated cohorts [15].

Our study has limitations. First, as noted, our sample size precluded intensive cross-validation of the predictive accuracy of covariates for false-positive Xpert determinations. The AUC describes how well models can rank order cases and non-cases, but is not a function of the actual predicted probabilities. Second, our criteria for false detection of active TB (multiple negative reference cultures in the setting of a positive Xpert) would be strengthened by protocolised withholding of anti-TB medications. However, this would be ethically infeasible in a programmatic setting given the imperfect negative predictive value of Xpert and patient immunosuppression. Still, some proportion of "false-positive" Xpert results may have actually been true-positive, as has been noted in other studies from high HIV-burden settings [16, 17].

In conclusion, patients with history of prior TB are exceptional both for an elevated likelihood of harbouring nonviable organisms and an increased pretest probability of TB. Within this group, we found that Xpert results with low mean cycle thresholds (Xpert quantitation "high" or greater) are unlikely to be false-positive. Clinicians should consider quantitative cycle threshold in addition to RMP-resistance determination when interpreting Xpert results among retreatment TB suspects.

@ERSpublications

Xpert false-positivity may be common in recurrent TB. High Xpert quantitation result is unlikely to be false-positive http://ow.ly/Ilg0F

John Z. Metcalfe ${ }^{1}$, Salome Makumbirofa ${ }^{2}$, Beauty Makamure $^{2}$, Reggie Mutetwa $^{2}$, Renée A. Peñaloza ${ }^{1}$, Charles Sandy ${ }^{3}$, Wilbert Bara ${ }^{4}$, Stanley Mungofa ${ }^{4}$, Philip C. Hopewell ${ }^{1}$ and Peter Mason ${ }^{2,5}$

${ }^{1}$ Curry International Tuberculosis Centre, Division of Pulmonary and Critical Care Medicine, San Francisco General Hospital, University of California, San Francisco, CA, USA. ${ }^{2}$ Biomedical Research and Training Institute, Harare, Zimbabwe. ${ }^{3}$ National Tuberculosis Control Program, Harare, Zimbabwe. ${ }^{4}$ Harare City Health Department, Harare, Zimbabwe. ${ }^{5}$ University of Zimbabwe College of Health Sciences, Harare, Zimbabwe. 
Correspondence: John Z. Metcalfe, University of California, San Francisco, Division of Pulmonary and Critical Care Medicine, San Francisco General Hospital, 1001 Potrero Avenue, Rm 5K1, San Francisco, CA 94110-0111, USA.

E-mail: john.metcalfe@ucsf.edu

Received: Sept 232014 | Accepted after revision: Jan 052015 | First published online: March 182015

Support statement: This study received funding from U.S. Department of Health and Human Services National Institutes of Health (grant number: K23 AI094251), Trials of Excellence for Southern Africa Network, Robert Wood Johnson Foundation AMFDP Medical Faculty Development Award, and U.S. Department of Health and Human Services Centers for Disease Control and Prevention (grant number: U52/CCU900454). Funding information for this article has been deposited with FundRef

Conflict of interest: None declared.

Acknowledgements: We would like to thank Harare City Health Department (Harare, Zimbabwe) clinicians and staff for their support and assistance.

\section{References}

1 World Health Organization. Global Tuberculosis Report 2014. Geneva, WHO Press, 2014. www.who.int/tb/ publications/global_report/en/

2 Weyer K, Mirzayev F, Migliori GB, et al. Rapid molecular TB diagnosis: evidence, policy making and global implementation of Xpert MTB/RIF. Eur Respir J 2013; 42: 252-271.

3 Pantoja A, Fitzpatrick C, Vassall A, et al. Xpert MTB/RIF for diagnosis of tuberculosis and drug-resistant tuberculosis: a cost and affordability analysis. Eur Respir J 2013; 42: 708-720.

4 Steingart KR, Schiller I, Horne DJ, et al. Xpert ${ }^{\oplus} \mathrm{MTB} / \mathrm{RIF}$ assay for pulmonary tuberculosis and rifampicin resistance in adults. Cochrane Database Syst Rev 2014; 1: CD009593.

5 Kennedy N, Gillespie SH, Saruni AO, et al. Polymerase chain reaction for assessing treatment response in patients with pulmonary tuberculosis. J Infect Dis 1994; 170: 713-716.

6 Friedrich SO, Rachow A, Saathoff E, et al. Assessment of the sensitivity and specificity of Xpert MTB/RIF assay as an early sputum biomarker of response to tuberculosis treatment. Lancet Respir Med 2013; 1: 462-470.

7 Boyles TH, Hughes J, Cox V, et al. False-positive Xpert ${ }^{\circledast}$ MTB/RIF assays in previously treated patients: need for caution in interpreting results. Int J Tuberc Lung Dis 2014; 18: 876-878.

8 Kelly JD, Grace Lin SY, Barry PM, et al. Xpert MTB/RIF false detection of rifampin-resistant tuberculosis from prior infection. Am J Respir Crit Care Med 2014; 190: 1316-1318.

9 Boehme CC, Nabeta P, Hillemann D, et al. Rapid molecular detection of tuberculosis and rifampin resistance. N Engl J Med 2010; 363: 1005-1015.

10 Boehme CC, Nicol MP, Nabeta P, et al. Feasibility, diagnostic accuracy, and effectiveness of decentralised use of the Xpert MTB/RIF test for diagnosis of tuberculosis and multidrug resistance: a multicentre implementation study. Lancet 2011; 377: 1495-1505.

11 Nicol MP. Xpert MTB/RIF: monitoring response to tuberculosis treatment. Lancet Respir Med 2013; 1: 427-428.

12 World Health Organization. Noncommercial culture and drug-susceptibility testing methods for screening of patients at risk of multidrug resistant tuberculosis: policy statement. Geneva, WHO Press, 2011. http://whqlibdoc. who.int/publications/2011/9789241501620_eng.pdf

13 Martin L, Coronel J, Faulx D, et al. A field evaluation of the Hardy TB MODS kit for the rapid phenotypic diagnosis of tuberculosis and multi-drug resistant tuberculosis. PLoS One 2014; 9: e107258.

14 Dorman SE, Chihota VN, Lewis JJ, et al. Performance characteristics of the Cepheid Xpert MTB/RIF test in a tuberculosis prevalence survey. PLoS One 2012; 7: e43307.

15 Pencina MJ, D'Agostino RB, Pencina KM, et al. Interpreting incremental value of markers added to risk prediction models. Am J Epidemiol 2012; 176: 473-481.

16 Rachow A, Zumla A, Heinrich N, et al. Rapid and accurate detection of Mycobacterium tuberculosis in sputum samples by Cepheid Xpert MTB/RIF assay - a clinical validation study. PLoS One 2011; 6: e20458.

17 Theron G, Peter J, van Zyl-Smit R, et al. Evaluation of the Xpert MTB/RIF assay for the diagnosis of pulmonary tuberculosis in a high HIV prevalence setting. Am J Respir Crit Care Med 2011; 184: 132-140. 